\title{
Laser-Driven Programmable Metal Transfer in GMAW
}

\author{
Double-sided, laser-enhanced gas metal arc welding was studied \\ to obtain the desired droplet trajectory
}

\author{
BY S. J. CHEN, Y. Z. JIA, J. XIAO, AND W. H. HUANG
}

\begin{abstract}
Conventional pulsed laser-enhanced gas metal arc welding (GMAW) employs a single fiber laser focused and aimed on the droplet neck position to produce a laser recoil force and thus ensure the droplet detachment despite the amperage of the welding current. One drop per laser pulse metal transfer is obtained, and the droplet deflects away from the wire axis along the laser incident direction. This implies that the droplet trajectory may also be controlled if the direction of the laser recoil force can be adjusted. Such a controllability is expected to bring an entirely new capability to the GMAW process: active control on the weld beam geometry. To this end, double-sided, laser-enhanced GMAW was proposed and experimentally verified in this paper. The two lasers were symmetrically positioned, and both aimed at the droplet neck. The laser pulse peak power, duration, and pulse phase of the two lasers can all be programmed to regulate the laser recoil forces. The metal transfer under twin laser irradiations (same laser pulses and phases) was first verified. Then the effectiveness on controlling the droplet trajectory of three proposed control strategies - peak power matching, peak width matching, and phase matching of the two lasers - were evaluated. The results showed laser peak power matching is optimal for obtaining desired droplet trajectory. Since the laser can be easily controlled in real time, the transfer frequency, droplet size, and trajectory can all be adjusted in real time, and the metal transfer evolves into programmable transfer.
\end{abstract}

\section{KEYWORDS}

- Double-Sided Laser Irradiation - Droplet Trajectory

- Programmable Metal Transfer • Gas Metal Arc Welding (GMAW)

\section{Introduction}

Gas metal arc welding (GMAW) is one of the most widely used welding processes in industrial manufacturing, as well as a basic process for wire arc additive manufacturing. However, conventional GMAW cannot accurately adjust the heat input and mass input to the base metal as desired, according to a specific application, due to the metal transfer mode generally coupled with the welding current. During the GMAW process, metal transfer has the greatest influence on process stability and welding quality. The desired drop spray transfer requires the welding current to exceed the spray transition current (Refs. 1-3). Achieving drop spray transfer at any reasonable low current used to be thought of as the ultimate goal of metal transfer control. Furthermore, the mindset is that drop spray transfer is better when approximately along the wire axial direction.

Successful efforts on active control of the metal transfer during past decades can be categorized two ways (Refs. 4-19): 1) modulating the welding current waveform, which can precisely optimize and control the instantaneous arc force and accumulated energy inside the droplet to improve the metal transfer process, such as surface tension transfer, pulsed GMAW, and the active droplet oscillation method; and 2) introducing an external detaching force, such as cold metal transfer, in a mechanical way; the bypass arc GMAW and magnetic field-assisted GMAW in an electromagnetic way; and ultrasonic-wave assisted GMAW and laserenhanced GMAW in a radiating way. Although a special welding current waveform may contribute to reducing the heat input and decreasing the spatter, it cannot decouple the metal transfer from the welding current. Applying an external detaching force is the only possible solution to achieve complete current-independent metal transfer.

The pulsed laser-enhanced GMAW process has indeed realized current-independent metal transfer under any reasonable low current (Ref. 20). The latest study on this topic adopted a pulsed laser that aimed the droplet to partially vaporize it, thus generating a recoil force to detach the droplet. However, since only a single laser was used, the droplet was always detached with a deflection away from the wire axial direction. Nonwire axial drop spray transfer is still not ideal because of the general mindset that the metal transfer should be ideally along the wire axial direction. In fact, slight droplet deflection would not compromise bead formation quality as long as the deflection angle is stable. Furthermore, it can be imagined whether the droplet deflection can be freely adjusted in a certain range/space in real time. Naturally, it will lead to a new milestone of metal transfer con- 


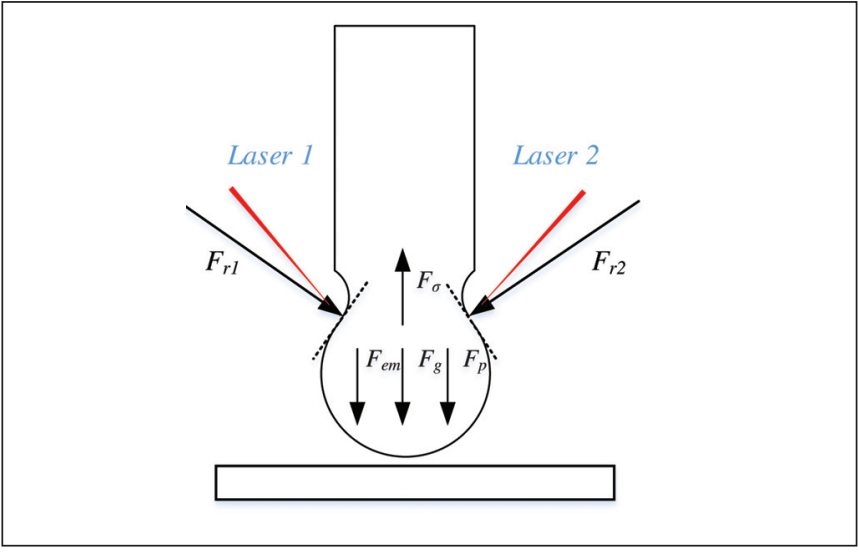

Fig. 1 - Principle of programmable droplet transfer in GMAW.

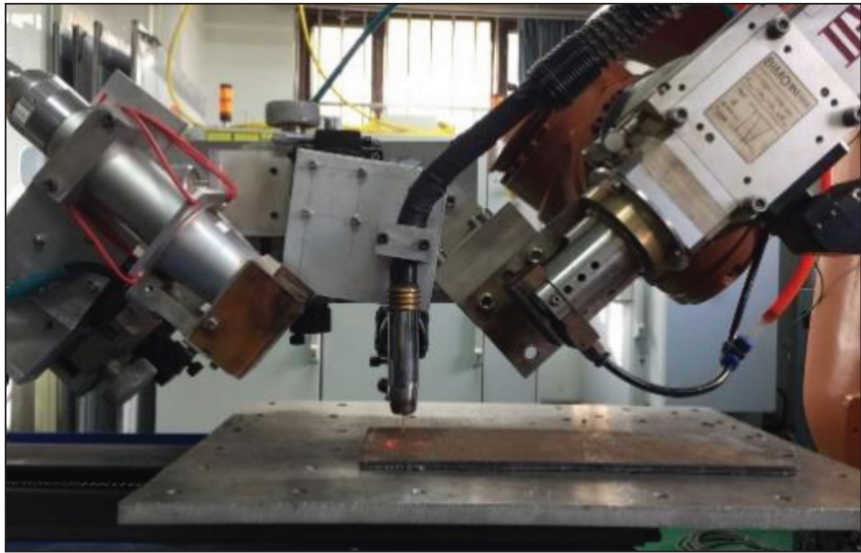

Fig. 3 - Installation of laser heads.

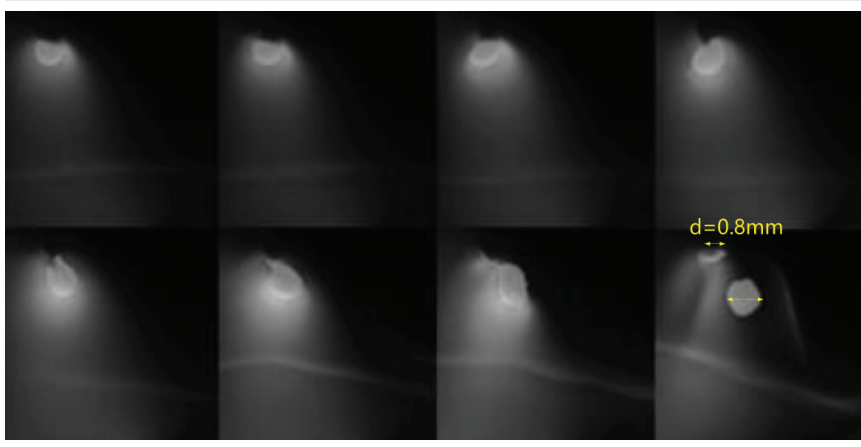

Fig. 5 - Single-pulsed, laser-enhanced metal transfer.

trol: The weld pool geometry and thus the weld bead formation could be actively controlled. In this sense, the ultimate goal of metal transfer control evolves from currentindependent transfer to more advanced programmable transfer.

Multilaser-enhanced GMAW may be a practical implementation to obtain the expected programmable transfer since the laser peak power and width can both be adjusted in real time. Subsequently, the magnitude/direction of the resultant laser recoil force exerting on the droplet changes and the droplet would be driven to different directions. To this end, this paper will experimentally verify the proposed laser-driven programming metal transfer. Restricted by the

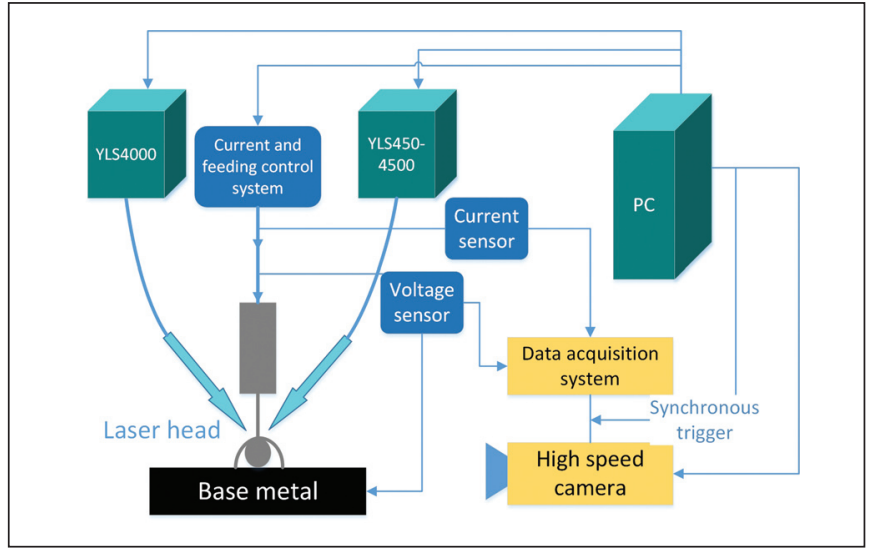

Fig. 2 - Sketch of the experimental system.

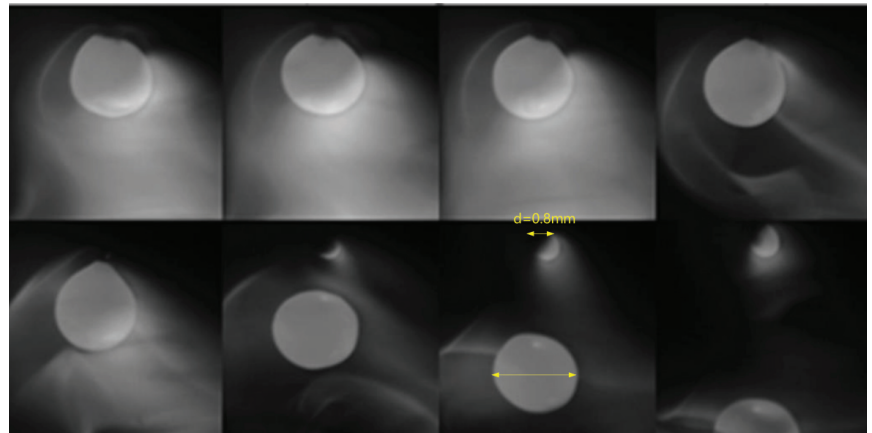

Fig. 4 - Typical metal transfer without laser.
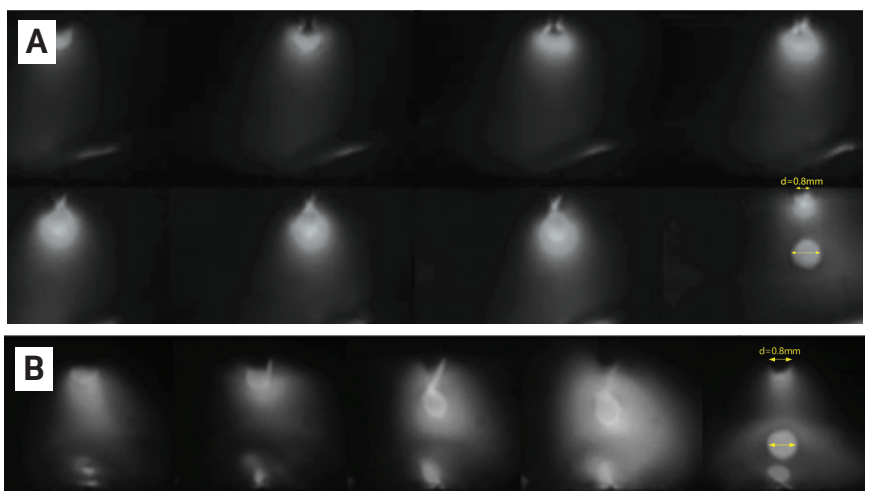

Fig. 6 - Typical metal transfer with double-sided even laser irradiation: A - Experiment 3, 25-Hz double-sided laser irradiation; B - Experiment 4, 150-Hz double-sided laser irradiation.

laser equipment, only double-sided, dual laser-driven metal transfer was studied. The proposed programmable metal transfer behavior will be verified and analyzed.

\section{Principle of Laser-Driven Programmable Metal Transfer}

Normally, the droplet pendant at the wire tip is affected by the following forces: the droplet gravity $F_{g}$, surface tension $F_{\sigma}$, electromagnetic force $F_{e m}$, and plasma dragging force $F_{p}$ as shown in Fig. 1. The gravity, electromagnetic force, and plasma flow force contributed to detaching the droplet from the wire tip, while the surface tension hindered it. The two lasers were 


\begin{tabular}{|c|c|c|c|c|c|c|c|}
\hline No. & $\begin{array}{c}\text { Current } \\
(\mathrm{I} / \mathrm{A})\end{array}$ & $\begin{array}{c}\text { Pulse } \\
\text { Frequency }\end{array}$ & $\begin{array}{l}\text { Peak Power of } \\
\text { Laser } 1(P / k W)\end{array}$ & $\begin{array}{l}\text { Peak Power of } \\
\text { Laser } 2(P / k W)\end{array}$ & $\begin{array}{l}\text { Pulse Width of } \\
\text { Laser } 1 \\
\text { (t/ms) }\end{array}$ & $\begin{array}{l}\text { Pulse Width of } \\
\text { Laser } 2 \\
\text { (t/ms) }\end{array}$ & $\begin{array}{l}\text { Phase Difference } \\
\qquad(\Delta t / \mathrm{ms})\end{array}$ \\
\hline 1 & 80 & 25 & 0 & 0 & 0 & 0 & - \\
\hline 2 & 80 & 25 & 1.5 & 0 & 4 & 0 & - \\
\hline 3 & 80 & 25 & 1.5 & 1.5 & 4 & 4 & - \\
\hline 4 & 80 & 150 & 1.5 & 1.5 & 2 & 2 & - \\
\hline 5 & 80 & 25 & 0.8 & 1.5 & 4 & 4 & - \\
\hline 6 & 80 & 25 & 1.0 & 1.5 & 4 & 4 & - \\
\hline 7 & 80 & 25 & 1.2 & 1.5 & 4 & 4 & - \\
\hline 8 & 80 & 25 & 1.8 & 1.5 & 4 & 4 & - \\
\hline 9 & 80 & 25 & 2.0 & 1.5 & 4 & 4 & - \\
\hline 10 & 80 & 25 & 2.2 & 1.5 & 4 & 4 & - \\
\hline 11 & 80 & 25 & 1.5 & 1.5 & 5 & 3 & - \\
\hline 12 & 80 & 25 & 1.5 & 1.5 & 6 & 2 & - \\
\hline 13 & 80 & 25 & 1.5 & 1.5 & 7 & 1 & - \\
\hline 14 & 80 & 25 & 1.5 & 1.5 & 4 & 4 & 1 \\
\hline 15 & 80 & 25 & 1.5 & 1.5 & 4 & 4 & 2 \\
\hline 16 & 80 & 25 & 1.5 & 1.5 & 4 & 4 & 3 \\
\hline
\end{tabular}

distributed to irradiate the droplet neck position symmetrically and produce two recoil forces, $F_{r 1}$ and $F_{r 2}$, which would play the primary role in detaching the droplet instead of the electromagnetic force. One may notice that the direction of laser recoil force is not exactly in the line of the laser beam, but experimentally verified in the normal of the very local irradiated surface (Ref. 20). The wire axial component of the recoil forces would elongate the droplet toward the weld pool, and the wire radial component of the $F_{r 1}$ and $F_{r 2}$ would balance each other's amplitude, thus changing the direction of the droplet deflection. It is reasonable to infer the droplet would be detached approximately without deflection from the wire if the two symmetrically positioned laser beams were the same pulse parameters. There may be three possible ways to further control the droplet trajectory, as follows:

1) Laser peak power match: use different peak power but the same pulse width and frequency, so the resultant radial laser recoil force may swing to the right or left.

2) Laser peak width match: use the same laser peak power and frequency but different pulse peak durations. In this case, the droplet is expected to deflect to the short-duration laser's side.

3) Laser pulse phase match: use the same peak power, same frequency, and same peak width, but different pulse rising moments. This method may also have the potential to drive the droplet into controllable waving.

By changing the laser pulse parameters of the two lasers, the two recoil forces would change and the droplet trajectory may change correspondingly. This is fundamental to obtaining the one droplet per laser pulse (ODPP) transfer that can be programmed offline or online. Finally, the GMAW process would possess the ability to determine when and where to detach each droplet.

\section{Experiment Setup}

The experimental system is composed of the welding cell, laser cell, high-speed camera, and data acquisition system, as shown in Fig. 2 . The welding power source works in constant current mode. As shown in Fig. 3, to the left is an IPGYLS4000 laser with a maximum peak power of $4 \mathrm{~kW}$, and to the right is a QCW450-4500 pulsed laser with a maximum peak power of $4.5 \mathrm{~kW}$, while the maximum average power is $0.45 \mathrm{~kW}$. Both lasers were focused to a point with a diameter of about $0.2 \mathrm{~mm}$, and the laser incident angle was about 45 deg. To evaluate the process, the welding current and voltage were measured during the experiments. The frequency of the high-speed camera and data acquisition were all set to $3 \mathrm{kHz}$. The wire was ER70S- 6 with a $0.8 \mathrm{~mm}$ diameter. The welding current waveform, wire feed speed, laser pulse waveform, high-speed camera system, and data acquisition system were all controlled by the PC-based central controller. The welding experiments were conducted as beadon-plate welding with $3 \mathrm{~mm} / \mathrm{s}$ travel speed and $15 \mathrm{~L} / \mathrm{min}$ 


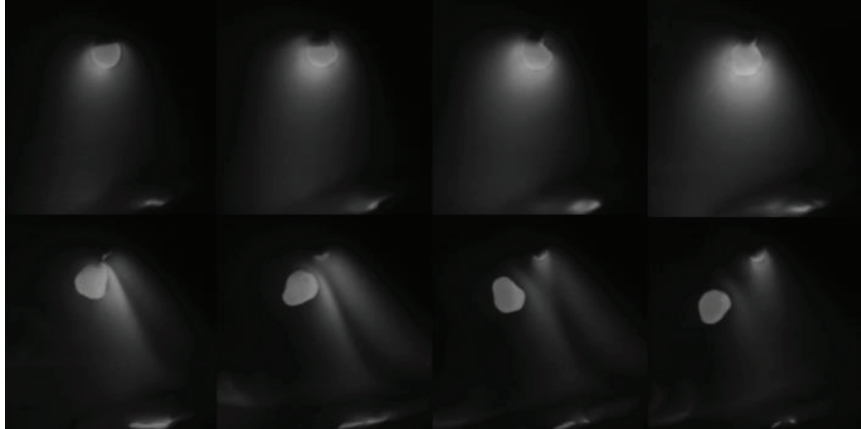

Fig. 7-Typical metal transfer in Experiment 5 with left laser peak power of $0.8 \mathrm{~kW}$.

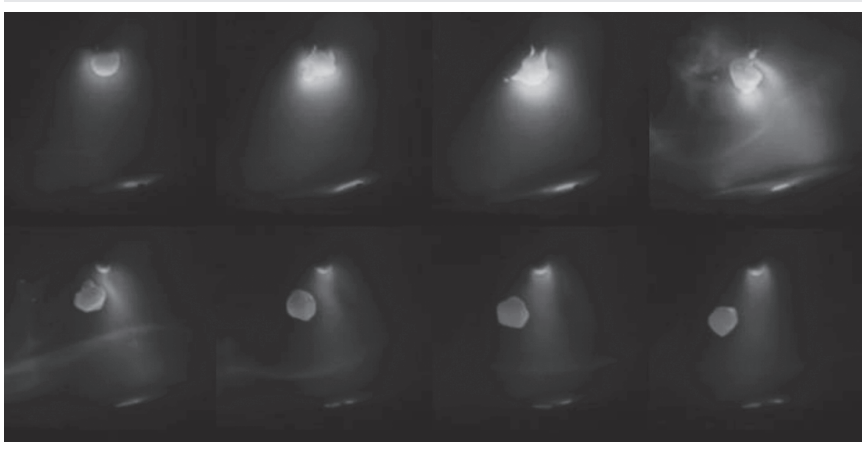

Fig. 9 - Typical metal transfer in Experiment 7 with left laser peak power of $1.2 \mathrm{~kW}$.

pure argon shielding gas. The base metal was mild steel. The distance from the contact tip to the workpiece was set at 15 $\mathrm{mm}$, and arc length was controlled at approximately $8 \mathrm{~mm}$. The key parameters of the double-sided, laser-driven programmable metal transfer are listed as follows: 1) laser pulse frequency, which mainly determines the metal transfer frequency/detached droplet size; 2) laser peak power, which mainly affects the magnitude of the laser recoil force; 3 ) laser pulse width, which mainly affects the time of recoil force exerting on the droplet; and 4) laser phase difference, defined as the time offset between the double-sided laser pulses. The welding parameters are shown in Table 1 . The two lasers will be referred to as the left one and the right one for convenience.

\section{Results and Discussion}

\section{Effect of Laser Irradiation}

To provide a clearer description of the double-sided, laser-controlled programmable droplet transfer, the metal transfer behavior of low welding current without laser (Experiment 1) and with single-sided pulsed laser irradiation (Experiment 2) were first analyzed for later comparison. When the welding current was $80 \mathrm{~A}$, the droplet was mainly detached by its gravity. It can be seen from Fig. 4 that the metal transfer is of the drop globular mode, and the droplet diameter is times larger than that of the wire. The transfer frequency is only about $4 \mathrm{~Hz}$. Such a lower transfer frequency tends to result in discontinuous and irregular bead formation, and makes the arc unstable. After a single-pulsed

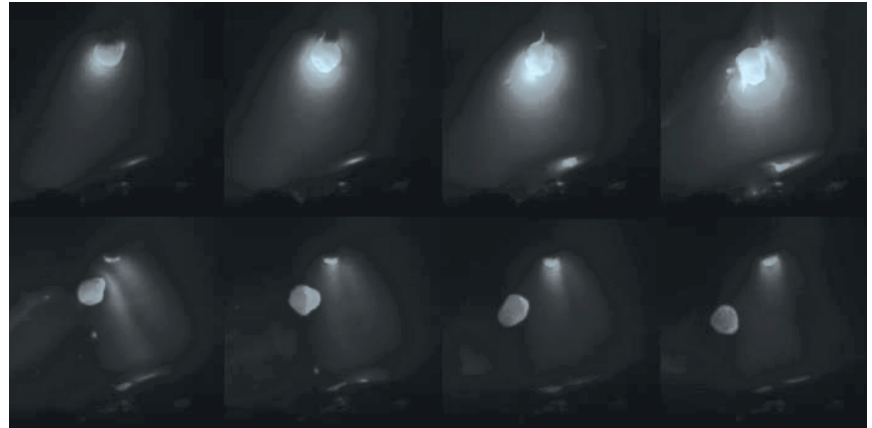

Fig. 8 - Typical metal transfer in Experiment 6 with left laser peak power of $1.0 \mathrm{~kW}$.

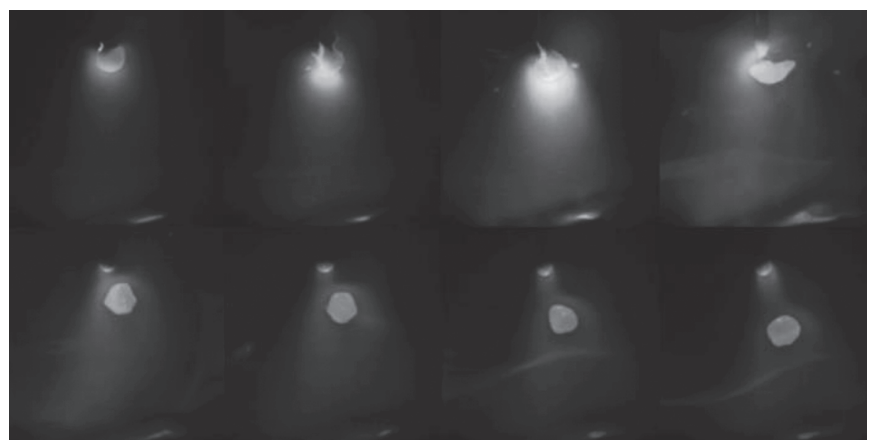

Fig. 10 - Typical metal transfer in Experiment 8 with left laser peak power of $1.8 \mathrm{~kW}$.

laser irradiates the area of the solid-liquid interface of the droplet, as shown in Fig. 5, the droplet is forced to be detached from the welding wire by the laser recoil force while the flying direction of the droplet deviates from the wire axis. The laser pulse frequency was $25 \mathrm{~Hz}$, and the pulse peak power was $1.5 \mathrm{~kW}$. The metal transfer frequency equaled the laser pulse frequency. One droplet per pulse (ODPP) transfer was obtained.

In addition, the double-sided, pulsed laser-driven metal transfer (the two lasers are with the same pulse parameters) was also verified and analyzed (Experiments 3 and 4). Unlike the single laser-driven metal transfer, two even laser recoil forces, marked as $F_{r 1}$ and $F_{r 2}$, were produced to drive the droplet deformation and motion. Since the two lasers were the same peak power/duration/frequency, emitted synchronously and arrayed symmetrically, the droplet deflection was thus avoided and the metal transfer frequency was significantly increased up to $150 \mathrm{~Hz}$ under an 80-A current. As shown in Fig. 6, the detached droplet diameter was only $0.85 \mathrm{~mm}$, approximately equal to the wire diameter. The droplet neck/wire tip was shaped to a "pencil tip" profile, similar to what usually exists in high-current-induced drop streaming transfer. If the droplet trajectory can be further controlled, the merging point of droplets into the molten pool and the morphology of the molten pool can be actively controlled. The key to changing the droplet trajectory is to modulate the laser recoil forces.

\section{Laser Peak Power Matching}

Given the laser spot size, the laser peak power determines its power density, which determines the magnitude of 


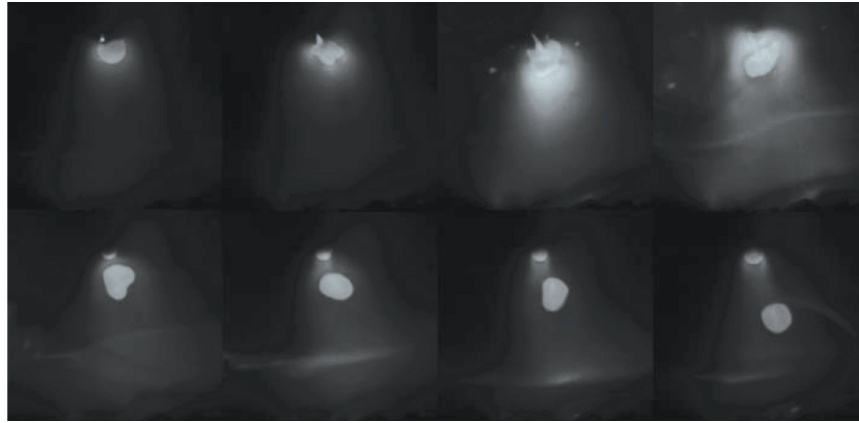

Fig. $11-$ Typical metal transfer in Experiment 9 with left laser peak power of $2.0 \mathrm{~kW}$.

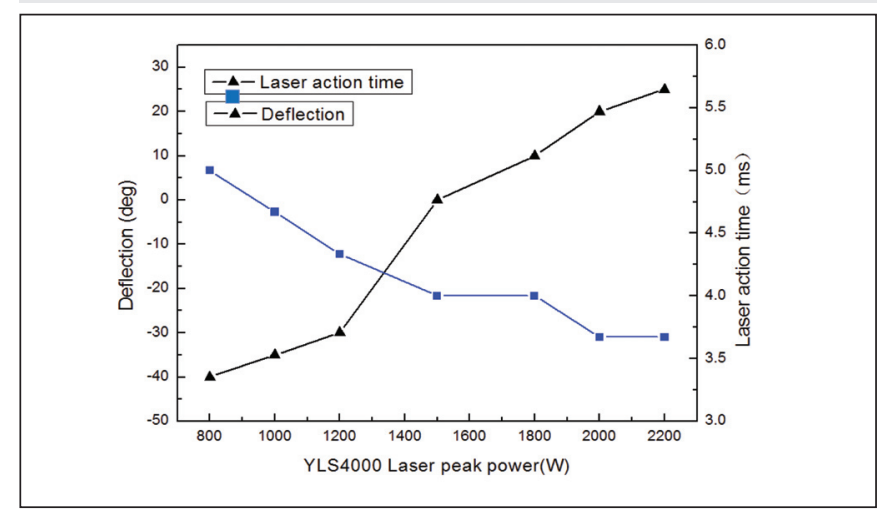

Fig. 13 - Effect of different laser peak powers on droplet deflection; the right laser is set to a constant $1.5 \mathrm{~kW}$.

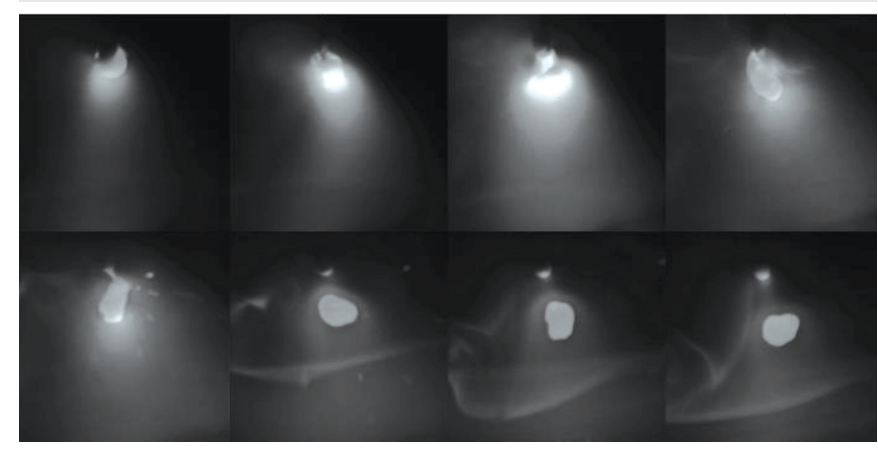

Fig. 15 - Typical metal transfer in Experiment 11.

the recoil force acting on the droplet. The laser pulse frequency in Experiments 5-10 was fixed at $25 \mathrm{~Hz}$. The laser peak duration was fixed at $4 \mathrm{~ms}$, just half of that used for single laser-driven experiments. The peak power of the right laser was fixed at $1.5 \mathrm{~kW}$. The two laser pulse waveforms were synchronized with no phase offset. It was verified that the resulting laser recoil force was not strong enough to detach the droplet in one pulse cycle when the left laser peak power was as low as $0.5 \mathrm{~kW}$. The minimum left laser peak power enabling to produce ODPP transfer with double-sided laser irradiation and only 4-ms laser peak duration was tested to be $0.8 \mathrm{~kW}$ under the welding conditions in this research. Thereby, Experiments 5-10 used left laser peak power of $0.8,1.0,1.2,1.8,2.0$, and $2.2 \mathrm{~kW}$, respectively. The results of Figs. 5 and 6 will be referred to for comparison.

Since the laser recoil force increased with the laser power density, the laser recoil force of the left laser $F_{r 1}$ in Experi-

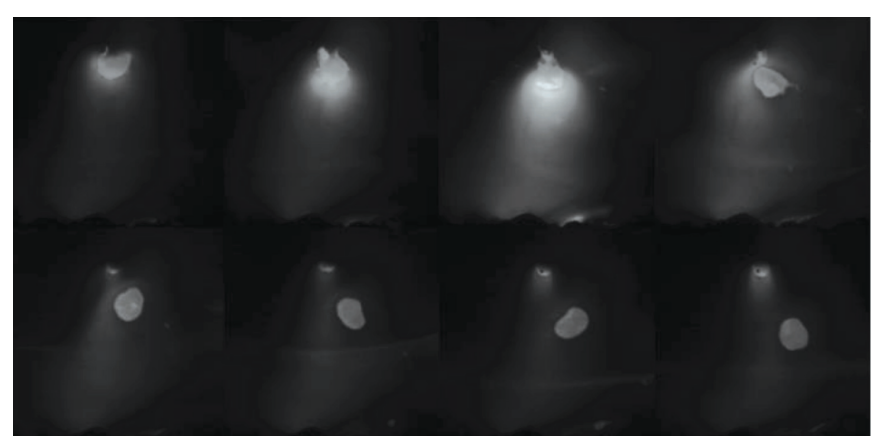

Fig. 12 - Typical metal transfer in Experiment 10 with left laser peak power of $2.2 \mathrm{~kW}$.

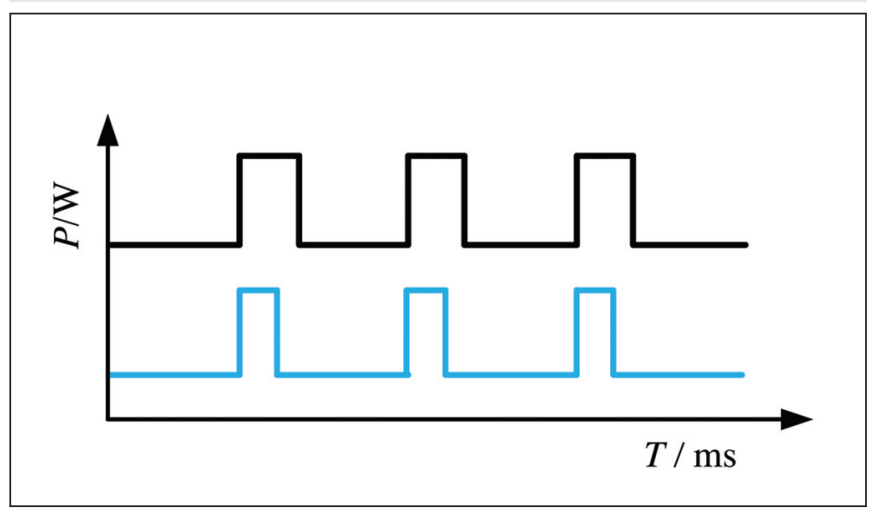

Fig. 14 - Principle of matching different pulse widths.

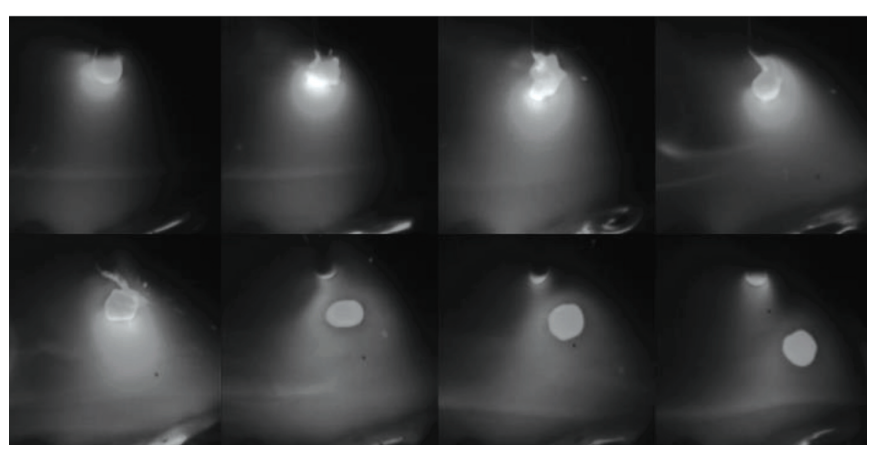

Fig. 16 - Typical metal transfer in Experiment 12.

ments 5-10 was increased gradually from smaller than $F_{r 2}$ to bigger than that. The droplet deflection was expected to change correspondingly. When the pulse peak power ranges from 0.8 to $1.5 \mathrm{~kW}$, as shown in Figs. 7-9, the droplet deflection gets slighter. With the increase in the left laser peak power, the flight direction of the droplet deviated from the wire axial direction but also gradually got closer to it. Since the two lasers are symmetrically aligned, metal transfer with desired deflections to the right side can be easily produced by mirroring the laser pulse parameters of the two lasers.

Experiments 8-10 further increased the peak power of the left laser to verify if the droplet would still be pushed to the right. When the left laser power was $1.8-2.2 \mathrm{~kW}$, as shown in Figs. 10-12, the droplet detached with deflection to the right side, just as expected. When the lasers irradiated the solid-liquid interface of the droplet, due to the intense recoil force and its cutting/digging effect, the droplet ap- 


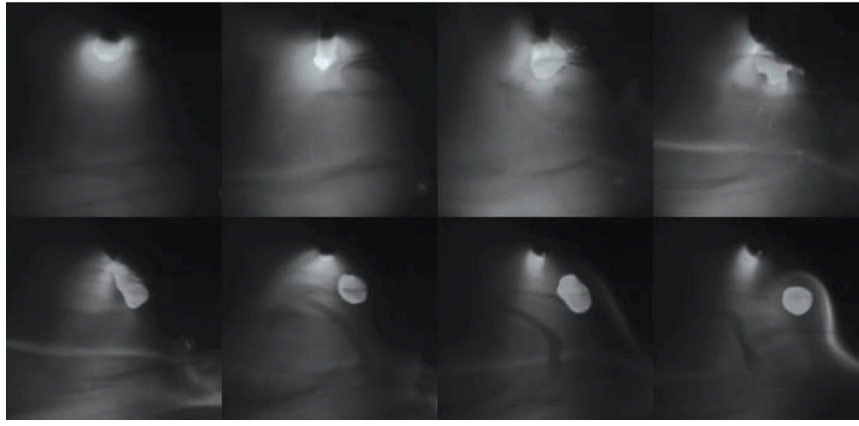

Fig. 17- Typical metal transfer in Experiment 13.

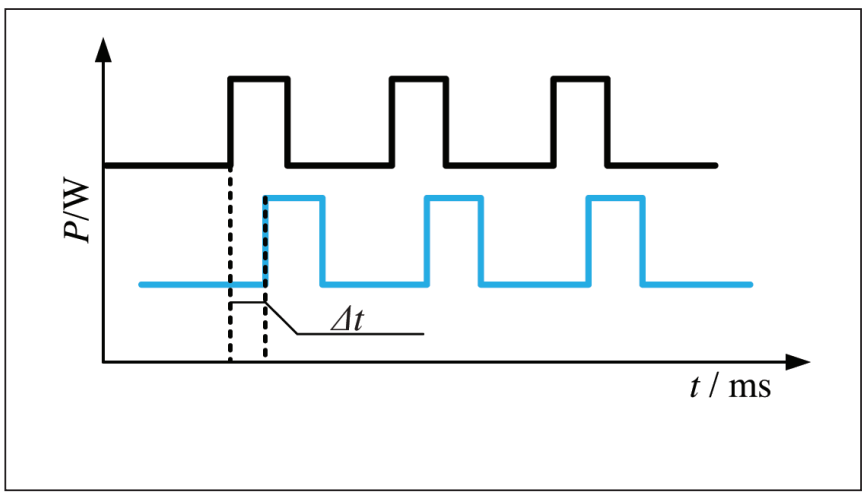

Fig. 19 - Illustration of double-sided laser phase match.

peared as an obvious forced necking, and the neck speed of the double side depended on the laser recoil force. Unbalanced necking speed results in droplet deflection. With the increased laser peak power of the laser, the droplet was easier to get detached from the welding wire tip. The droplet deflections and laser detaching time in Experiments 5-10 are measured and shown in Fig. 13. The actual laser detaching time on the droplet was reduced from 5 to $3.7 \mathrm{~ms}$ with a laser peak power increase from 0.8 to $2.2 \mathrm{~kW}$.

\section{Pulse Laser Width Matching}

In fact, as can be seen from Fig. 6 , when the pulse frequency and pulse peak power are the same, the laser peak power density of both sides are equal, and the radial recoil forces on the droplet are balanced with each other in the same pulse width of $4 \mathrm{~ms}$. The resultant force is in the wireaxial direction and detaches the droplet in the wire axial direction. When the single-sided laser pulse width was $8 \mathrm{~ms}$, as shown in Fig. 5, the droplet detached with a deflection. As shown in Fig. 14, matching the pulse width of the two lasers may also enable active control of droplet deflection. Experiments 11-13 were conducted to verify the effect of pulse width match. The sum of the two lasers' peak width was fixed at $8 \mathrm{~ms}$. As shown in Figs. 15-17, with the increase of the left laser pulse width and the decrease of the right laser pulse width, the digging/cutting time of each laser changes, resulting in an increased droplet deflection angle, as measured and shown in Fig. 18. Different deflection angles can be obtained with different laser peak width matches, and the merging point of the droplet into the molten pool can thus be controlled.

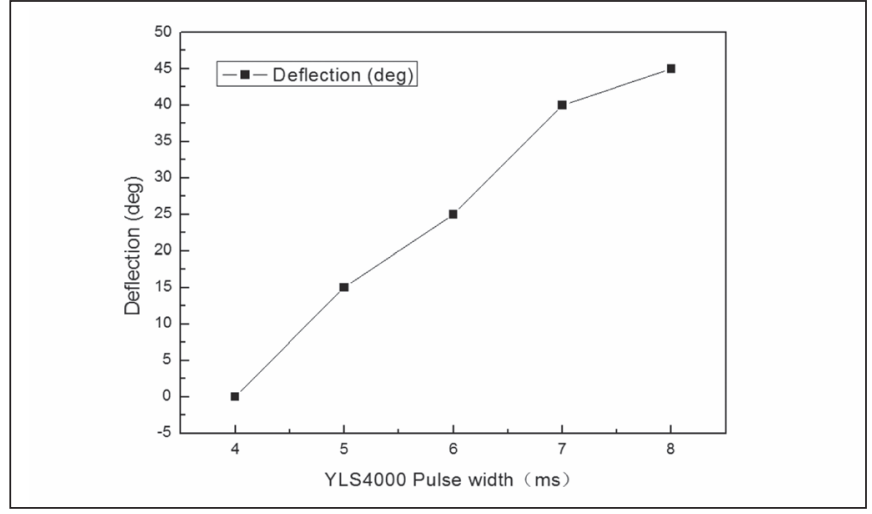

Fig. 18 - Effects of matching different pulse widths.
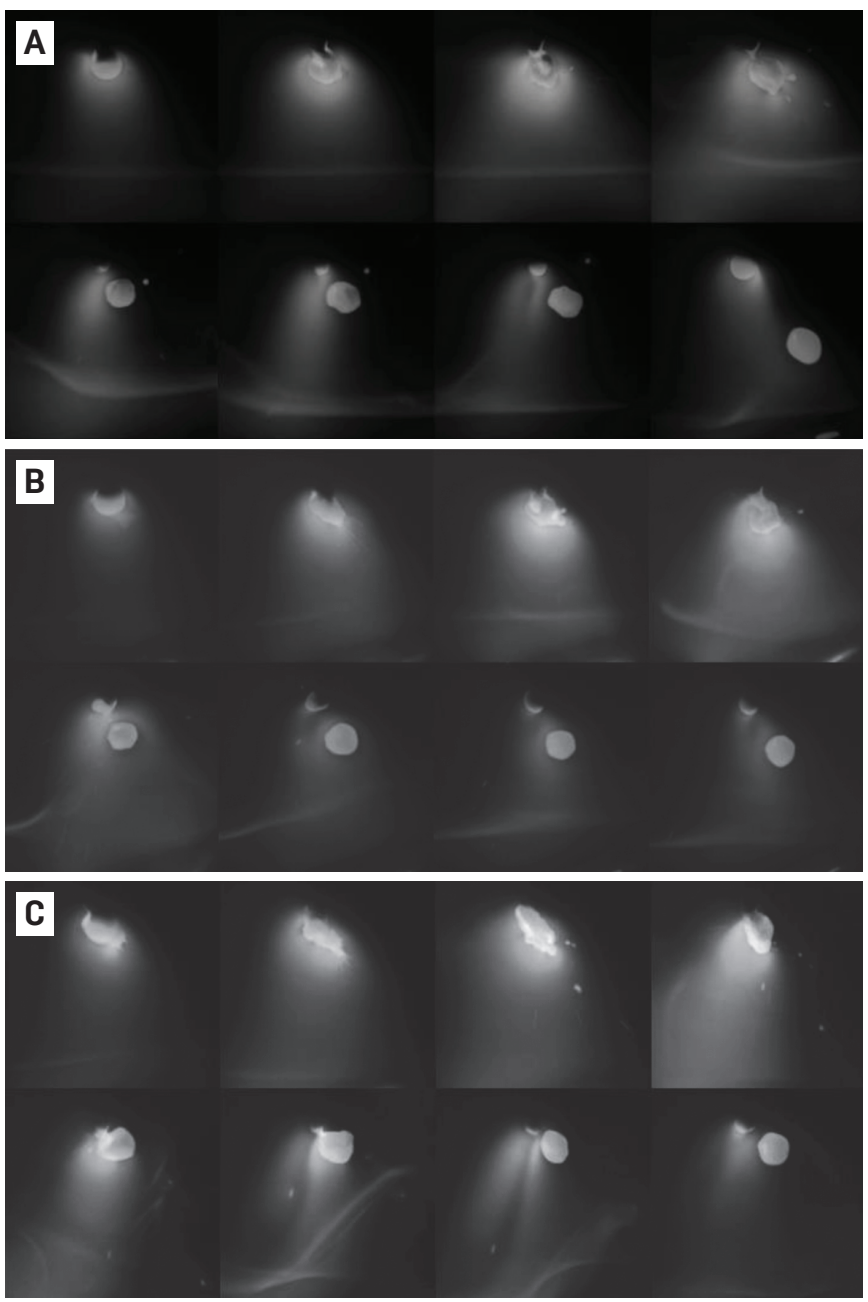

Fig. $20-$ Typical metal transfer in Experiments 14-16: $A-\Delta t$ $=1 \mathrm{~ms} ; \mathrm{B}-\Delta t=2 \mathrm{~ms} ; \mathrm{C}-\Delta t=3 \mathrm{~ms}$.

\section{Laser Peak Phase Matching}

The effect of double-sided laser phase matching was verified by Experiments 14-16 in this section. The peak powers of the two lasers were both fixed at $1.5 \mathrm{~kW}$, and the peak widths were the same at $4 \mathrm{~ms}$. The laser phase difference $\Delta t$ (as shown in Fig. 19), which is the time sequence of the 

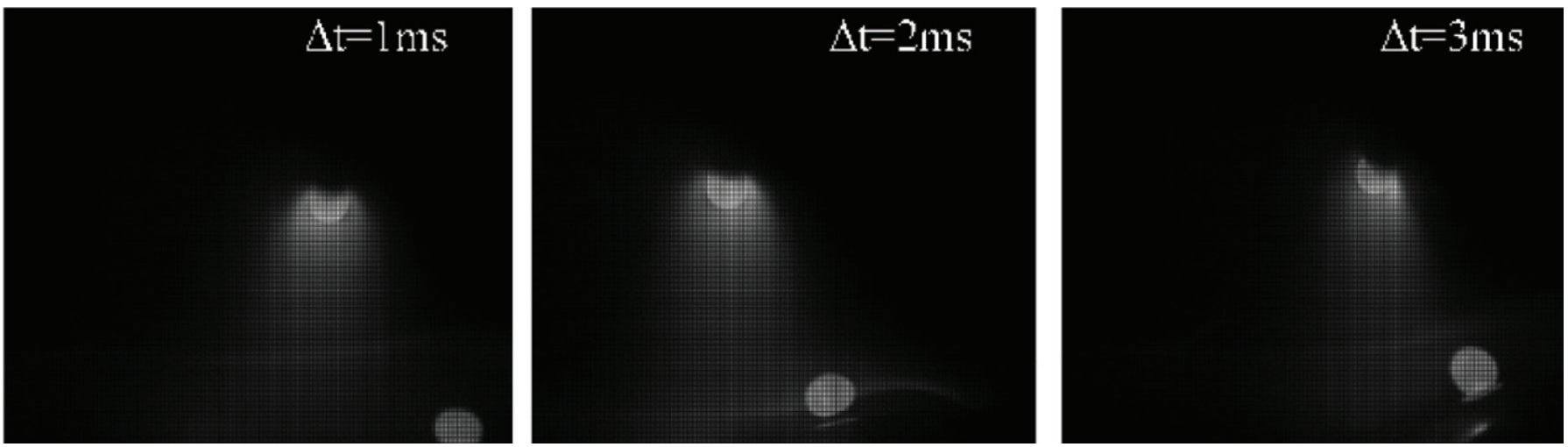

Fig. 21 - Final droplet deflection in Experiments 14-16.

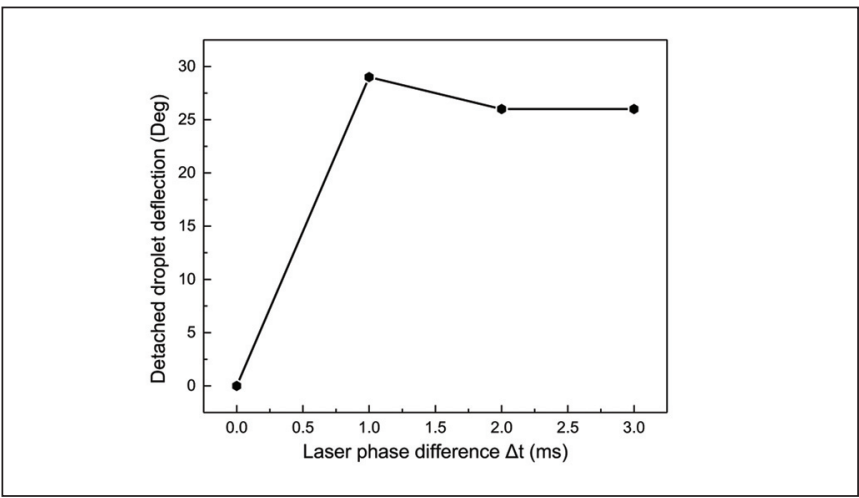

Fig. 22 - Measured droplet deflections in Experiments 3 and 14-16.

pulsed lasers, determined the effecting time sequence of the laser recoil force. Figure $20 \mathrm{~A}-\mathrm{C}$ shows the typical metal transfer in Experiments 14-16. Figure 21 shows the frames of actual final deflections at the merging into pool moment, and the deflections are measured and shown in Fig. 22.

It can be seen that the droplet deflection did not noticeably change with the increase of the laser phase difference. Particularly, for only $1 \mathrm{~ms}$ slight $\Delta t$, the increment of droplet deflection tended to be significant compared with that of 0 $\mathrm{ms} \Delta t$. As shown in Fig. $6 \mathrm{~A}$, with the same $4 \mathrm{~ms}$ pulse width and $0 \mathrm{~ms} \Delta t$, the droplet detached along the wire axially. As shown in Fig. 20A, the first frame was at the peak start moment of the left laser, and the second frame was at the peak start moment of the right laser. There is a little brighter reflection at the right neck position of the droplet, as can be seen in Frame 2 of Fig. 20A. At this moment, the droplet was already driven to an initial deflection by the left laser. However, the $F_{r 2}$, the laser recoil force on the right side, didn't show a capability to effectively restrict the droplet deflection as may be expected. The reason lies in the directions and distribution of the two laser recoil forces. As illustrated in Fig. 23, Frame 2 of Fig. 20A was picked for analysis. Since it has been verified in our previous study that the direction of laser recoil force is of the normal of the irradiated local surface despite the laser incident angle, the state of the two laser recoil forces can be illustrated as Fig. 23 shows. In this case, the $F_{r 2}$ had more proportions of radial component, but did not push against the droplet and restrict the deflection because the two recoil forces were forming a twisting couple.

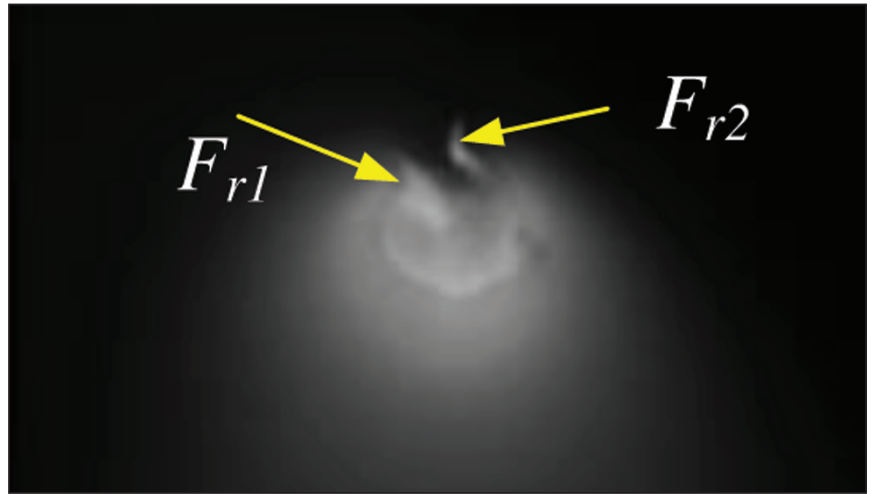

Fig. 23 - Illustration of the laser recoil force couple with $1 \mathrm{~ms}$ $\Delta t$.

That is why only $1 \mathrm{~ms} \Delta t$ would lead to a significantly increased droplet deflection.

One may also notice from Fig. 20 that the detaching moment of different $\Delta t$ changed. Longer $\Delta t$ resulted in later detach because it reduced the effective co-detaching time of the two laser pulses. That is to say, the resultant detaching ability of the two lasers was weakened with a longer phase match, and using a phase match method will not facilitate the desired programmable transfer.

\section{Conclusion}

1) The double-sided laser irradiation method has shown its significantly improved capability on enhancing metal transfer. When the two lasers are symmetrically positioned and irradiated for the droplet with exactly the same laser pulse parameters and zero pulse phase difference, the droplet deflection that would always be associated with the single laser irradiation process can be avoided. Additionally, the metal transfer frequency can be increased up to $150 \mathrm{~Hz}$ measured with the welding wire and conditions in this paper.

2) Laser peak power match and peak width match can both lead to effective control of the droplet deflection in a range determined by using only one laser irradiation. Since the laser pulse parameters can be easily programmed online or offline, the evolution of the GMAW process is approaching the new milestone featured by programmable metal 
transfer. Since the laser peak power has a much wider adjusting range, the peak power match strategy is supposed to be optimal for programmable metal transfer control.

3) Laser pulse phase match strategy is verified to not be effective in regulating the droplet deflection. As long as there is a phase difference between the double-sided laser pulse, even as narrow as $1 \mathrm{~ms}$, the droplet will be driven into deflection, and the deflection doesn't change significantly with the increase of laser pulse phase difference.

\section{Acknowledgments}

This work is supported by the Natural Science Foundation of China under grant No. 51505009 and 51575133, and the opening fund from the State Key Laboratory of Advanced Welding and Joining, Harbin Institute of Technology, Harbin, China.

\section{References}

1) Iordachescu, D., and Quintino, L. 2008. Steps toward a new classification of metal transfer in gas metal arc welding. Journal of Materials Processing Technology 202 (1-3): 391-397. DOI: 10.1016/j.jmatprotec.2007.08.081

2) Rhee, S., and Kannatey-Asibu, E. 1991. Analysis of arc pressure effect on metal transfer in gas metal arc welding. Journal of Physics D: Applied Physics 24(8): 5068-5075. DOI: 10.1063/ 1.349014

3) Kim, Y. S., and Eagar, T. W. 1993. Analysis of metal transfer in gas metal arc welding. Welding Journal 72(6): 269-s to 278-s.

4) Deruntz, B. D. 2003. Assessing the benefits of surface tension transfer welding to industry. Journal of Industrial Technology 19(4): 2-8.

5) Acobsen, N. 1992. Monopulse investigation of droplet detachment in pulsed gas metal arc welding. Journal of Physics D: Applied Physics 25: 783-797.DOI: 10.1088/0022-3727/25/5/007

6) Zhu, M., Shi, Y., and Fan, D. 2015. Analysis and improvement of metal transfer behaviors in consumable double-electrode GMAW process. Journal of Manufacturing Science and Engineering 137(2): 011010.1-011010.5. DOI: 10.1115/1.4028636

7) Himmelbauer, K. 2005. The CMT process - A revolution in welding technology. IIW Doc XII-1875-05, 20-27, IIW.

8) Chan, Y. L., et al. 2014. Impacts of external longitudinal magnetic field on arc plasma and droplet during short-circuit GMAW.
International Journal of Advanced Manufacturing Technology 70(912): 1543-1553. DOI: $10.1007 / \mathrm{s} 00170-013-5403-1$

9) Shi, Y., Liu, X., Zhang, Y. M., and Johnson, M. 2008. Analysis of metal transfer and correlated influences in dual-bypass GMAW of aluminum. Welding Journal 87(9): 229-s to 236-s.

10) Wu, Y., and Kovacevic, R. 2002. Mechanically assisted droplet transfer process in gas metal arc welding. Journal of Engineering Manufacturing 216: 555-564.

11) Zheng, B., and Kovacevic, R. 2001. A novel control approach for the droplet detachment in rapid prototyping by 3D welding. Journal of Manufacturing Science and Engineering 123: 348-355.

12) Fan, C. L., Yang, C. L., Lin, S. B., and Fan, Y. Y. 2013. Arc characteristics of ultrasonic wave-assisted GMAW. Welding Journal 92(12): 375-s to 380-s. DOI: 10.1007/s00170-017-1414-7

13) Fan, Y. Y., Yang, C. L., Lin, S. B., and Fan, C. L. 2012. Ultrasonic wave-assisted GMAW. Welding Journal 91(3): 91-s to 98-s.

14) Li, K. H., and Zhang, Y. M. 2008.Consumable doubleelectrode GMAW - Part 2: Monitoring, modeling, and control. Welding Journal 87(2): 44-s to 50-s.

15) Huang, Y., and Zhang, Y. M. 2010. Laser enhanced GMAW. Welding Journal 89(9): 181-s to 188-s.

16) Huang, Y., and Zhang, Y. M. 2011. Laser enhanced metal transfer - Part 1: System and observations. Welding Journal 90(10): 183-s to 190-s.

17) Huang Y., and Zhang Y. M. 2011. Laser enhanced metal transfer - Part 2: Analysis and influence factors. Welding Journal 90(11): 206-s to 210-s.

18) Zhang, Y. M, Li, G. E, and Kovacevic, R. 1998. Active metal transfer control by monitoring excited droplet oscillation. Welding Journal 77(9): 388-s to 395-s.

19) Zhang, Y. M, and Li, P. J. 2001. Modified active control of metal transfer and pulsed GMAW of titanium. Welding Journal 80(2): 54 -s to 61-s. DOI: 10.1034/j.1600-0692.2001.300107.x

20) Xiao, J., Chen, S. J., Zhang, G. J., and Zhang, Y. M. 2016. Current-independent metal transfer by using pulsed laser irradiation - Part 1: System and verification. Welding Journal 95(3): 93-s to 100 -s.

SHUJUN CHEN, YAZHOU JIA, WENHAO HUANG, and JUN XIAO (jun.xiao@bjut.edu.cn) are with the Engineering Research Center of Advanced Manufacturing Technology for Automotive Components, Ministry of Education, College of Mechanical Engineering and Applied Electronics, Beijing University of Technology, Beijing, China. XIAO is also with the State Key Laboratory of Advanced Welding and Joining, Harbin Institute of Technology, Harbin, China.

\section{Can We Talk?}

The Welding Journal staff encourages an exchange of ideas with you, our readers. If you'd like to ask a question, share an idea, or voice an opinion, you can call, write, email, or fax. Staff email addresses are listed below, along with a guide to help you interact with the right person.

Publisher/Editor Annette Alonso aalonso@aws.org

Managing Editor, Welding Journal Kristin Campbell kcampbell@aws.org,

Sr. Editor Cindy Weihl cweihl@aws.org

Associate Editor Katie Pacheco pacheco@aws.org
Education Editor Roline Pascal rpascal@aws.org

Peer Review Coord. Lea Owen Lea@aws.org

Managing Editor, Digital and Design Editor, Inspection Trends and Welding Journal en Español Carlos Guzman

cguzman@aws.org 